\title{
Targeted Elastin-like Fusion Proteins for Enhanced Imaging and Treatment of Non-Muscle Invasive Bladder Cancer
}

\author{
Matthew L. Nordland ${ }^{1}$, Craig J. Sweet ${ }^{2}$, Mollie Shinkle ${ }^{2}$, David H. Thompson ${ }^{3}$ \\ ${ }^{1}$ Purdue University School of Engineering, Department of Biomedical \\ Engineering, ${ }^{2}$ Indiana University School of Medicine.
}

\section{Background and Hypothesis:}

A recent study estimates 17,670 individuals will die from bladder cancer in the United States in 2019. With current imaging and treatment technology, bladder cancer is associated with high recurrence rates making it the highest lifetime treatment cost per patient. High expense is primarily due to the profound heterogeneity associated with bladder cancer necessitating repeated intervention. Traditional treatment of non-muscle invasive bladder (NMIBC) cancer following intravesical tumor resection often includes chemotherapy or immunostimulatory therapy via Bacillus Calmette-Guerin (BCG) infusions. Both lack specificity for cancerous tissue, leading to sub-optimal prognosis and unnecessary discomfort for patients. Furthermore, bladder cancer detection is limited in its precision. Methods such as CT/MRI urography and cystoscopies are unable to accurately detect tumors under 2 centimeters in diameter. Blue light cystoscopy, a gold standard detection method, is unable to accurately differentiate tumors from inflamed tissue. This leads to issues detecting aggressive carcinoma in situ. The goal of this project is to enhance tumor detection by fusing various targeting ligands to elastin-like peptides (ELP).

\section{Experimental Design or Project Methods:}

Using synthetic biology approaches, a library of ELP fusions were made and purified using a simplified organic extraction method. (Biomaterials Science 2018, 6 (4), 863-876) This resulted in exceptionally pure protein within a matter of hours. Contrast agents were covalently attached, and performance testing was done in murine bladder cancer cells.

\section{Results:}

Novel ELP fusions were successfully produced and sequences verified. Protein expression and purification yielded active targeted constructs which showed enhanced binding efficiency over non-targeted constructs. These ELP fusion proteins are effective transporters for rapid and preferential cargo entry into bladder cancer tissue.

\section{Conclusion and Potential Impact:}

Chemotherapeutic, immunostimulatory, and tumor imaging cargo are all bright candidates for targeted ELP fusion technology. Targeted ELP delivery may be a superior tool key to improving tumor visualization and treatment, leading to improved comfort and prognosis in patients with NMIBC. 